\title{
NONINVARIANCE OF AN APPROXIMATION PROPERTY FOR CLOSED SUBSETS OF RIEMANN SURFACES
}

\author{
BY \\ STEPHEN SCHEINBERG ${ }^{1}$
}

\begin{abstract}
A closed subset $E$ of an open Riemann surface $M$ is said to have the approximation property $\mathbb{Q}$ if each continuous function on $E$ which is analytic at all interior points of $E$ can be approximaed uniformly on $E$ by functions which are everywhere analytic on $M$. It is known that $\mathscr{Q}$ is a topological invariant (i.e., preserved by homeomorphisms of the pair $(M, E)$ ) when $M$ is of finite genus but not in general, not even for $C^{\infty}$ quasi-conformal automorphisms of $M$. The principal result of this paper is that $\mathscr{Q}$ is not invariant even under a real-analytic isotopy of quasi-conformal automorphisms (of a certain $M$ ). $M$ is constructed as the two-sheeted unbranched cover of the plane minus a certain discrete subset of the real axis, and the isotopy is induced by $(x+i y, t) \mapsto x+i t y$, for $t>0 ; E$ can be taken to be that portion of $M$ which lies over a horizontal strip.
\end{abstract}

Let $M$ be an open Riemann surface and $E$ be a closed subset of $M$. Denote by $A(E)$ the collection of continuous functions on $E$ which are analytic on the interior of $E$. Say that $E$ has property $Q$ in $M$ if each element of $A(E)$ can be approximated uniformly on $E$ by functions which are analytic everywhere on $M$. By definition $\mathbb{Q}$ is a property of the pair $(M, E)$ and is a conformal invariant; that is, if one pair is related to another by an analytic homeomorphism, the both pairs have $\mathbb{Q}$ or neither one does. It is natural to ask whether $\mathbb{Q}$ is invariant for other types of equivalence. The famous theorem of Bishop and Mergelyan implies that $\mathbb{Q}$ is a topological invariant in case $E$ is compact, and the theorem of Arakelyan shows that $\mathbb{Q}$ is a toplogical invariant when $M$ is planar. By "topological invariant" I mean an invariant for the equivalence defined by homeomorphism of pairs. It is known [S] that $\mathscr{Q}$ is a topological invariant when $M$ is of finite genus but is not a topological invariant in general. In fact, $\mathcal{Q}$ is not an invariant even for the finer partition induced by this relation: call $(M, E)$ equivalent to $\left(M^{\prime}, E^{\prime}\right)$ if and only if $M$ is conformally equivalent to $M^{\prime}$ and there is a quasi-conformal homeomorphism of $M$ onto $M^{\prime}$ which carries $E$ onto $E^{\prime}[\mathbf{S}]$. When I showed him the known example illustrating this phenonmenon, Dennis Sullivan asked me whether the example could be improved to one in which $Q$ fails to be preserved by an isotopy. The intent of this article is to provide an affirmative answer by demonstrating that a real-analytic isotopy need not preserve $\mathcal{Q}$, even though it is "affine", being

\footnotetext{
Received by the editors November 8, 1979.

1980 Mathematics Subject Classification. Primary 30E10, 30F99.

${ }^{1}$ Partially supported by NSF Grant MCS77-01848. I am grateful to the Hebrew University, Jerusalem, and the Mathematical Institute, Oxford, for their gracious hospitality during my visit in the spring of 1978, when much of this work was done.
} 
definable in local coordinates by $(x+i y, t) \rightarrow x+i t y$ for $0<t<\infty$, and all the homeomorphisms of $M$ throughout the homotopy are quasi-conformal equivalences. The precise statement is given below as the theorem. Let us write " $E \in \mathbb{Q}$ " in place of " $E$ has property $Q$ in $M$ ".

THEOREM. There is a connected open Riemann surface $M$, a homotopy $U: M \times \mathbf{R}^{+}$ $\rightarrow M$, and two closed connected subsets $E^{+}$and $E^{-}$of $M$ such that the following hold, where $U_{t}(p)$ is written in place of $U(p, t)$.

(a) $M$ can be given as a two-sheeted unbranched cover of a plane region by $Z$ : $M \rightarrow C-\mathscr{D}$, where $\mathscr{D}$ is a discrete subset of the real axis, and the homotopy $U$ is induced by the affine plane homotopy $u: \mathbf{C} \times \mathbf{R}^{+} \rightarrow \mathbf{C}$ defined by $u(x+i y, t)=x+$ ity; that is, $Z(U(p, t))=u(Z(p), t)$ for all $p \in M$ and $t \in \mathbf{R}^{+}$.

(b) $U$ is real-analytic on $M \times \mathbf{R}^{+}$.

(c) Each $U_{t}$ is a real-analytic quasi-conformal automorphism of $M$.

(d) $U_{1}$ is the identity of $M$.

(e) As $t \rightarrow 1$ the distortion $\left|\partial U_{t} / \partial \bar{Z}\right|\left|\partial U_{t} / \partial Z\right|^{-1} \rightarrow 0$ uniformly on $M$.

(f) $\left\{t: U_{t}\left(E^{+}\right) \in \mathbb{Q}\right\}=(0,1]$.

(g) $\left\{t: U_{t}\left(E^{-}\right) \in \mathbb{Q}\right\}=(0,1)$.

Because of the relation between $U$ and $u$ one may call $U$ or $U_{t}$ "affine". For $t$ near 1 the maps $U_{t}$ are as close to conformal and as close to the identity as may be desired; yet the slight movement of $E^{+}$to $U_{1+e}\left(E^{+}\right)$destroys $\mathscr{Q}$ and the slight movement of $E^{-}$to $U_{1-\varepsilon}\left(E^{-}\right)$creates $Q$.

The proof of the theorem will be along the following lines. A locally finite collection of closed intervals $J$ will be selected in $\mathbf{R}$ according to certain technical requirements. $M$ will be formed as follows: two copies of $\mathbf{C}$ minus all the $J$ 's will be joined in the standard way along corresponding slits $J$, leaving out the set $\mathscr{D}$ consisting of all the endpoints of the $J$ 's. The natural projection $Z: M \rightarrow C-\mathscr{D}$ exhibits $M$ as a two-sheeted unbranched covering of a plane region. The homotopy defined on $\mathbf{C} \times \mathbf{R}^{+}$by $u(x+i y, t)=x+$ ity transfers via $Z$ to a homotopy defined on each component of $Z^{-1}(V) \times \mathbf{R}^{+}$, where $V$ is any vertical strip in $\mathbf{C}-\mathscr{D}$ whose projection on the $y$-axis is $\mathbf{R}, \mathbf{R}^{+}$, or $\mathbf{R}^{-}$. These homotopies agree whenever there is an overlap; so they define a homotopy $U$ on $M$. Assertions (a)-(e) will follow immediately.

Define $S_{\lambda}$ to be the strip $\{x+i y:|y| \leqslant \lambda\}$ and $S_{\lambda}^{+}$(resp., $S_{\lambda}^{-}$) to be the intersection of $S_{\lambda}$ with the closed right (resp., left) half-plane. Define $E_{\lambda}^{ \pm}=$ $Z^{-1}\left(S_{\lambda}{ }^{ \pm}\right)$. The intervals $J$ will be arranged in $\mathbf{R}$ in such a fashion that for a certain $\lambda_{0}>0$ none of the sets $S_{\lambda}^{+}$for $\lambda>\lambda_{0}$ (resp., $S_{\lambda}^{-}$for $\lambda>\lambda_{0}$ ) supports a nontrivial bounded analytic function which vanishes on $S_{\lambda}^{+} \cap \mathscr{D}$ (resp., $\left.S_{\lambda}^{-} \cap \mathscr{D}\right)$ ). This will imply that $\mathbb{Q}$ fails for the corresponding $E_{\lambda}^{+}$(resp., $E_{\lambda}^{-}$). Because $U_{t}\left(E_{\lambda}^{+}\right)=E_{t \lambda}{ }^{ \pm}$, this will mean that one-half of (f) and (g), namely with " $\subseteq$ " in place of " $=$ ", will be true for $E^{+}=E_{\lambda_{0}}^{+}$and $E^{-}=E_{\lambda_{0}}^{-}$.

The proof of the other half of (f) and (g) is technically more difficult and will require the bulk of the work. These ideas will be involved. Fix one $E_{\lambda}^{+}, \lambda<\lambda_{0}$, or one $E_{\lambda}^{-}, \lambda<\lambda_{0}$, and call it $E$. Because of the spatial arrangement of $\mathscr{D}$ there will 
exist a sequence of meromorphic functions $H_{n}$ on $\mathrm{C}$ each of which has a zero or a pole at each point of $\mathscr{D}$ and nowhere else and each of which is small on a large bounded set $X_{n}$, is large on a co-compact subset $Y_{n}$ of $S_{\lambda}{ }^{ \pm}$, and is nearly 1 on a vertical strip $\sigma_{n}$ which separates $X_{n}$ from $Y_{n}$. Multiplying $H_{n}$ by an exponential function and extracting a square root, we obtain an analytic function $\pi_{n}$ on $M$ which separates these four sets: $Z^{-1}\left(X_{n}\right)$, the two components of $Z^{-1}\left(\sigma_{n}\right)$, and $Z^{-1}\left(Y_{n}\right) . E-\cup Z^{-1}\left(\sigma_{n}\right)$ consists of separated pieces of finite genus; so if $f \in$ $A(E)$, there is an analytic $\Phi$ on $M$ such that $g=f-\Phi$ is very small on $E-$ $\cup Z^{-1}\left(\sigma_{n}\right) . g$ can be approximated by $\sum g_{n}$, where $g_{n}$ is $C^{1}$ on $E^{\circ} \cup Z^{-1}\left(X_{n}\right)$, is supported in $Z^{-1}\left(\sigma_{n}\right)$, and has small $\bar{Z}$-derivative. Because $\pi_{n}$ separates the four sets indicated above, $g_{n}$ can be written $g_{n}=\tilde{g}_{n} \circ \pi_{n}$, where $\tilde{g}_{n}$ is (essentially) a smooth function of compact support in $\mathbf{C}$ having small $\bar{z}$-derivative. Because of this property of $\tilde{g}_{n}$ and of the nature of $\pi_{n}\left(Z^{-1}\left(X_{n} \cup \sigma_{n} \cup Y_{n}\right)\right), \tilde{g}_{n}$ can be approximated reasonably well by a rational function $k_{n}$. Thus, $k_{n} \circ \pi_{n}$ approximates $g_{n}$ on $E \cup Z^{-1}\left(X_{n}\right)$. The poles of $k_{n} \circ \pi_{n}$ in $M$ can be removed by a multiplier without essentially altering the goodness of the approximation to $g_{n}$. The resulting analytic functions $\psi_{n}$ on $M$ can be summed to an analytic function $\Psi$, because $Z^{-1}\left(X_{n}\right) \uparrow M$, and thus $\Phi+\Psi$ will approximate $f$.

The reader acquainted with [S] will recognize a similarity in spirit between the above scheme for showing $E \in \mathbb{Q}$ and the method used in $\$ 11$ of [S]. However, the method of $[\mathbf{S}]$ is technically simpler in several respects. For example, the projection $\pi$ of the surface in [S] served to separate all the components of all the $\pi^{-1}\left(\sigma_{n}\right)$ at once, and it was not necessary to allow singularities to arise in intermediate steps for later removal. The punctures in the surface of $[\mathbf{S}]$ allowed the existence of enough globally analytic functions but prevented the existence of an isotopy. The surface $M$ of this article is punctured in order to allow construction of the separating functions $\pi_{n}$; since the punctures lie over the real axis, they do not interfere with the isotopy. I thank Ted Gamelin for asking me whether a related result utilized an iteration of some sort. This remark prompted me to look for an approximation of $g=f-\Phi$ by means of separate approximations of "pieces" of $g$; this in turn opened up the possiblity of individual separating functions $\pi_{n}$ for each "piece". And I thank Dennis Sullivan for raising the question which this paper answers.

The rest of the paper is devoted to a proof of the theorem. Most of the technical aspects are gathered into manageable or convenient aggregates and termed lemmas or corollaries.

LEMMA 1 (a). If $|z| \leqslant \frac{1}{2}$, then $|\log (1+z)-z| \leqslant|z| / 2$.

(b) If $|w| \leqslant 1$, then $\left|e^{w}-1\right| \leqslant(e-1)|w| \leqslant 2|w|$.

(c) If $\Sigma\left|a_{n}\right| \leqslant \frac{1}{2}$, then $\left|\Pi\left(1+a_{n}\right)-1\right| \leqslant 3 \Sigma\left|a_{n}\right|$.

Proof. (a) and (b) are well known and follow readily from easy manipulations of Taylor series. Assume $\Sigma\left|a_{n}\right| \leqslant \frac{1}{2}$. From (a) it follows that $\left|\log \Pi\left(1+a_{n}\right)-\Sigma a_{n}\right|$ $\leqslant \frac{1}{2} \Sigma\left|a_{n}\right|$; so $\left|\log \Pi\left(1+a_{n}\right)\right| \leqslant \frac{3}{2} \Sigma\left|a_{n}\right| \leqslant \frac{3}{4}$. Now an application of (b) yields (c). 
LeMma 2. For $0<\lambda<\infty$ and complex $z_{0}$ and $z$ define

$$
\tau\left(z ; z_{0}, \lambda\right)=\left(a^{z}-a^{z_{0}}\right) /\left(a^{z}+a^{\bar{z}_{0}}\right),
$$

where $a=\exp (\pi / 2 \lambda)$. Suppose $0<\lambda<\pi(2 \log 2)^{-1}$ and $\left|x-x_{0}\right|>1$, where $x=$ $\operatorname{Re} z$ and $x_{0}=\operatorname{Re} z_{0}$. Let $\omega=\operatorname{signum}\left(x-x_{0}\right)$. Then

$$
\left|1-\omega \tau\left(z, z_{0}, \lambda\right)^{ \pm 1}\right| \leqslant 4 a^{-\left|x-x_{0}\right|}=4 \min \left(a^{x} \cdot a^{-x_{0}}, a^{x_{0}} \cdot a^{-x}\right) .
$$

Proof. In case $x \geqslant x_{0}+1$ compute

$$
\left|1-\tau^{+1}\right|=\left|\frac{a^{z_{0}}+a^{\bar{z}_{0}}}{a^{z}+a^{\bar{z}_{0}}}\right|=a^{x_{0}-x}\left|\frac{1+a^{\bar{z}_{0}-z_{0}}}{1+a^{\bar{z}_{0}-z}}\right|<a^{x_{0}-x} \frac{2}{1-a^{x_{0}-x}}<4 a^{x_{0}-x},
$$

because $\left|a^{\bar{z}_{0}-z_{0}}\right|=1$ and $a^{x_{0}-x} \leqslant a^{-1}<\frac{1}{2}$. The computations for $\tau^{-1}$ and for the case $x \leqslant x_{0}-1$ are very similar.

LEMMA 3. If $x_{n}$ is a sequence of distinct real numbers such that $\left|x_{n}\right| \rightarrow \infty$ and $0<\lambda<\infty$, the following are equivalent.

(a) There exists a nonzero bounded analytic function on $S_{\lambda}$ which vanishes on $X=\left\{x_{n}: n \geqslant 1\right\}$.

(b) On $S_{\lambda}^{+}$there is a nonzero bounded analytic function which vanishes on $X \cap S_{\lambda}^{+}$, and the analogous statement holds for $S_{\lambda}^{-}$.

(c) $\sum a^{-\left|x_{n}\right|}<\infty$, where $a=\exp (\pi / 2 \lambda)$.

(d) $\Pi_{n} \operatorname{sgn}\left(x_{n}\right)\left(a^{x_{n}}-a^{z}\right) /\left(a^{x_{n}}+a^{z}\right)$ converges normally on the plane to a function which is analytic on $S_{2 \lambda}^{\circ}$ (the interior of $S_{2 \lambda}$ ), is bounded by 1 on $S_{\lambda}$, and vanishes in $S_{2 \lambda}$ precisely on $X$.

Proof. The conformal map $w=\varphi(z)=\exp (\pi z / 2 \lambda)$ carries $S_{\lambda}$ to the closed right half-plane minus 0 and takes $X$ to a positive sequence $\left\{w_{n}\right\}$ which clusters only at 0 and /or $\infty$. The Blaschke condition for a real sequence $w_{n}$ tending to 0 (resp. $\infty$ ) in the right half-plane is easily seen to be $\sum w_{n}<\infty$ (resp., $\sum w_{n}^{-1}<\infty$ ). Because a convergent Blaschke product on the open half-plane is analytic on the closed half-plane minus the cluster set of its zeros, (a) is equivalent to (c). Trivially, (a) implies (b). The above mapping $w=\varphi(z)$ (resp., $w=1 / \varphi(z))$ sends $S_{\lambda}^{-}$(resp., $S_{\lambda}^{+}$) onto the half-disc $\{w:|w| \leqslant 1, \operatorname{Re} w>0\}-\{0\}$, which contains the disc $\Delta=\{w$ : $\left.\left|w-\frac{1}{2}\right|<\frac{1}{2}\right\}$. So (b) implies the Blaschke condition for the real sequence $w_{n}=$ $\varphi\left(x_{n}\right) \rightarrow 0$ (resp., $w_{n}=1 / \varphi\left(x_{n}\right) \rightarrow 0$ ) in $\Delta$, which implies (c). Recall that a sequence, series, or product of meromorphic functions $f_{n}$ is said to converge normally on a region $R$ if for each compact subset $K$ of $R$ all the functions $f_{n}$ are analytic on $K$ for $n \geqslant N(K)$ and the sequence, series, or product of the $f_{n}$ for $n \geqslant N(K)$ converges uniformly on $K$. Lemma 2 and simple observations about $\tau\left(z ; x_{n}, \lambda\right)$ reveal that (c) implies (d). Finally, (d) trivially implies (a).

LEMMA 4. There exist sequences $s_{n}, t_{n}, K_{n}$, and $N_{n}$ of positive integers such that

(1) $1 \leqslant s_{1}<t_{1}<s_{2}<t_{2}<\cdots$ and $1 \leqslant K_{1}<N_{1}<K_{2}<N_{2}<\cdots$,

(2) $2^{-t_{n} / n} \sum_{1}^{n} 2^{s_{j}} N_{j} \rightarrow 0$ as $n \rightarrow \infty$; in particular, $t_{n}-s_{n} \rightarrow \infty$,

(3) $16^{t_{n}} \sum_{n+1}^{\infty} 4^{-s_{j}}\left(K_{j}+4^{-s_{j} / j} N_{j}\right) \rightarrow 0$ as $n \rightarrow \infty$; in particular, $s_{n+1}-t_{n} \rightarrow \infty$ and $\sum_{1}^{\infty} 4^{-s_{n}}\left(K_{n}+4^{-s_{n} / n} N_{n}\right)<\infty$, and

(4) $\sum 4^{-s_{n}} N_{n}=\infty=\sum 4^{-s_{n}} 4^{e s_{n}} K_{n}$ for every $\varepsilon>0$. 
Proof. Define three sequences of integers by the recursion: $r_{1}=1, s_{n}=n r_{n}$, $t_{n}=n\left(3 s_{n}+n\right), r_{n+1}=2 t_{n}+n$. It is easy to see that $r_{n}$ and $s_{n}$ are strictly increasing with $n$ and that $s_{n+1}>n r_{n+1}>t_{n}>s_{n}$. Put $N_{n}=4^{s_{n}}$ and $K_{n}=N_{n} 4^{-s_{n} / n}$ $=N_{n} 4^{-r_{n}}=4^{s_{n}-r_{n}}=4^{(n-1) r_{n}}$. (1) and (4) are immediate.

Each of the sums $\Sigma_{1}^{n}$ in (2) and $\sum_{n+1}^{\infty}$ in (3) is readily seen to be a sum of distinct powers of 2. Therefore, each sum is at most twice its largest term. For the sum $\Sigma_{1}^{n}$ in (2) this estimate is $2 \cdot 2^{s_{n}} N_{n}=2 \cdot 2^{3 s_{n}}=2 \cdot 2^{t_{n} / n} \cdot 2^{-n}$, and (2) follows. For the sum $\sum_{n+1}^{\infty}$ in (3) the estimate is $2 \cdot 4^{-s_{n+1}} \cdot 2 K_{n+1}=4 \cdot 4^{n r_{n+1}-s_{n+1}}=4 \cdot 4^{-r_{n+1}}=4$. $4^{-2 t_{n}} \cdot 4^{-n}$, and (3) follows.

Let us turn now to the specification of $M, U, E^{+}$, and $E^{-}$. Put $\lambda_{0}=\pi(4 \log 2)^{-1}$; any value could serve for $\lambda_{0}$, but this one is convenient because $\exp \left(\pi / 2 \lambda_{0}\right)=4$. Select $s_{n}, t_{n}, K_{n}$, and $N_{n}$ according to Lemma 4 . For each $n>0$ select $K_{n}$ disjoint closed subintervals of the real interval $\left(s_{n}, s_{n}+1\right)$ and $N_{n}$ disjoint closed subintervals of $\left(-s_{n}-1,-s_{n}\right)$; refer to each of these tiny intervals as $J$. Consider the intervals $J$ as slits in the plane and join two copies of the plane slit by all the $J$ 's in the standard fashion by joining the upper edge of each $J$ in each plane with the lower edge of the same $J$ in the other plane. Call the resulting surface $\bar{M}$ and let $\bar{\pi}$ : $\bar{M} \rightarrow \mathrm{C}$ be the natural projection of $\bar{M}$ onto C. $\bar{M}$ is exhibited as a branched two-sheeted cover of $\mathbf{C}$. Let $B \subseteq \bar{M}$ be the set of branch points of this covering; that is $\beta \in B$ if and only if $d \bar{\pi}(\beta)=0$ if and only if $\bar{\pi}(\beta)$ is an endpoint of one of the intervals $J$. Now define $M=\bar{M}-B, Z=\left.\bar{\pi}\right|_{M}$, and $\mathscr{D}=\bar{\pi}(B)=$ the set of endpoints of the $J$ 's. $Z: M \rightarrow C-\mathscr{D}$ realizes $M$ as a two-sheeted unbranched cover of the region $C-\mathscr{D}$. Denote by $E_{\lambda}^{ \pm}$the sets $Z^{-1}\left(S_{\lambda}^{ \pm}\right)$in $M$ and put $E^{+}=E_{\lambda_{0}}^{+}$and $E^{-}=E_{\lambda_{0}}^{-}$.

As previously indicated, $U$ will be induced by the plane isotopy $u(x+i y, t)=x$ $+i t y, t>0$, in this manner. $M$ is covered by open sets $T$ for which $\left.Z\right|_{T}$ is a homeomorphism and $Z(T)=I \times I^{\prime}$, where $I$ is an open interval of $\mathbf{R}$ and $I^{\prime}=\mathbf{R}$ or $\mathbf{R}^{+}$or $\mathbf{R}^{-}$. $I^{\prime}$ can equal $\mathbf{R}$ precisely when $I$ is disjoint from $\mathscr{D}$. For each such $T$ define a homotopy $U_{T}: T \times \mathbf{R}^{+} \rightarrow T$ by $U_{T}(p, t)=\left(\left.Z\right|_{T}\right)^{-1} u(Z(p), t)$. It is clear that if $T_{1} \cap T_{2} \neq \varnothing$, then for all $p \in T_{1} \cap T_{2}$ and all $t>0, U_{T_{1}}(p, t)=U_{T_{2}}(p, t)$ $=U_{T_{1} \cap T_{2}}(p, t)$. Therefore, we may define $U(p, t)=U_{T}(p, t)$ for any $T$ which contains $p$ and for all $t>0$. Because $Z$ is a local coordinate at every point of $M$ and is an analytic homeomorphism on each $T$, it is immediate from the definition of $U$ and elementary properties of $u$ that (a)-(e) hold.

Because $u_{t}\left(S_{\lambda}^{ \pm}\right)=S_{t \lambda}^{ \pm}$it is immediate that $U_{t}\left(E_{\lambda}^{ \pm}\right)=E_{t \lambda}^{ \pm}$. So (f) and (g) are equivalent to the following statements:

(f') $E_{\lambda}^{+} \in Q$ if and only if $\lambda \leqslant \lambda_{0}$.

(g') $E_{\lambda}^{-} \in \mathbb{Q}$ if and only if $\lambda<\lambda_{0}$.

The "only if" parts of ( $\left.\mathrm{f}^{\prime}\right)$ and $\left(\mathrm{g}^{\prime}\right)$ are proved as follows. Fix $\lambda \geqslant \lambda_{0}$ and define $a=a(\lambda)=\exp (\pi / 2 \lambda) . a\left(\lambda_{0}\right)=4$, and $a(\lambda)=4^{1-e}$ for some $\varepsilon>0$ when $\lambda>\lambda_{0}$. For $0 \leqslant s<x<s+1$ and $a \leqslant 4$ we find $a^{-x}>4^{-1} a^{-s}$ and $4^{-x}>4^{-1} 4^{-s}$. From Lemma 3 and from part (4) of Lemma 4 it then follows that if $\varphi$ is a bounded analytic function on $S_{\lambda}^{-}$for $\lambda \geqslant \lambda_{0}$ (or on $S_{\lambda}^{+}$for $\lambda>\lambda_{0}$ ) which vanishes at every point of $\mathscr{D} \cap S_{\lambda}^{-}$(or $\mathscr{D} \cap S_{\lambda}^{+}$), then $\varphi$ vanishes identically. The following lemma 
then yields that the corresponding sets $E_{\lambda}^{ \pm}=Z^{-1}\left(S_{\lambda}^{ \pm}\right)$in $M$ do not belong to $Q$. Special cases of this lemma were used in [S].

LEMMA 5. Suppose $\pi_{1}: M_{1} \rightarrow R$ is a two-sheeted branched cover of a connected open subset $R$ of $C$ with branch set $B_{1} \subseteq M_{1}$. Let $B_{0} \subseteq B_{1}$; put $M_{2}=M_{1}-B_{0}$ and $\pi_{2}=\left.\pi_{1}\right|_{M_{2}}$. If $S$ is a subregion of $R$ such that $\bar{S} \neq R$ and 0 is the only bounded analytic function on $S$ which vanishes at all points of $\pi_{1}\left(B_{1}\right) \cap S$, then $\pi_{2}^{-1}(\bar{S})$, which is $\pi_{1}^{-1}(\bar{S})-B_{0}$, does not have property $\mathbb{Q}$ in $M_{2}$.

Proof. For any function $g$ on $\pi_{2}^{-1}(S)$, define $\Delta g$ on $S-\pi_{1}\left(B_{1}\right)$ by $\Delta g(z)=$ $\left(g\left(p_{1}\right)-g\left(p_{2}\right)\right)^{2}$, where $\left\{p_{1}, p_{2}\right\}=\pi_{2}^{-1}(z)$. (See [RS], where this idea is used.) $\Delta g$ is analytic on $S-\pi_{1}\left(B_{1}\right)$ whenever $g$ is analytic on $\pi_{2}^{-1}(S)$. If furthermore $g$ is bounded on $\pi_{2}^{-1}(S)$, Riemann's theorem on removable singularities implies that $g$ extends to a bounded analytic function on $\pi_{1}^{-1}(S) \subseteq M_{1}$ and that $\Delta g$ extends similarly to $S$. The extended $\Delta g$ vanishes at every point $z_{1} \in \pi_{1}\left(B_{1}\right) \cap S$, because as $z$ tends to $z_{1}$ the two points $p_{1}$ and $p_{2}$ coalesce to the single point of $B_{1}$ lying over $z_{1}$. By hypothesis $\Delta g$ must therefore vanish identically on $S$ whenever $g$ is a bounded analytic function on $\pi_{2}^{-1}(S)$.

Now choose $z_{0} \in R-\left(\bar{S} \cup \pi_{1}\left(B_{1}\right)\right)$ and let $\left\{p_{1}, p_{2}\right\}=\pi_{2}^{-1}\left(z_{0}\right)$. Select a meromorphic function $f$ on $M_{2}$ which has a pole at $p_{1}$ as its only singularity [BS]. Then $f$ is analytic on $\pi_{2}^{-1}(\bar{S})$ and yet $f$ cannot be approximated uniformly on $\pi_{2}^{-1}(\bar{S})$ by an analytic function $F$ on $M_{2}$. For if $F$ were analytic on $M_{2}$ and $|f-F|<1$ on $\pi_{2}^{-1}(\bar{S})$, the foregoing paragraph shows that $\Delta(f-F) \equiv 0$ on $S$. By uniqueness of analytic functions $\Delta(f-F) \equiv 0$ on $R-\left(\pi_{1}\left(B_{1}\right) \cup\left\{z_{0}\right\}\right)$. However, this is contradicted by the fact that $f-F$ is bounded near $p_{2}$ and unbounded near $p_{1}$. So such an $F$ does not exist, and $\pi_{2}^{-1}(\bar{S})$ does not have property $Q$ in $M_{2}$.

The proof of the "if" parts of (f') and (g'), namely, that $E_{\lambda}^{+} \in Q$ for $0<\lambda \leqslant \lambda_{0}$ and that $E_{\lambda}^{-} \in \mathbb{Q}$ for $0<\lambda<\lambda_{0}$ will require the construction of certain auxiliary functions on $C$ and on $M$. Henceforth let $b$ be a variable ranging over $\mathscr{D}=\bar{\pi}(B)$ $=$ the set of endpoints of the intervals $J$, and let $s_{n}, t_{n}, K_{n}$, and $N_{n}$ be as in Lemma 4. Define functions $A_{n}, B_{n}, C_{n}$, and $D_{n}$ as follows, where for $b<0$ we let $k=k(b)$ be the unique integer such that $s_{k}<-b<s_{k}+1, \lambda_{b}=k \lambda_{0}(1+k)^{-1}$, and $a_{b}=$ $\exp \left(\pi / 2 \lambda_{b}\right)=4 \cdot 4^{1 / k}$.

$$
\begin{aligned}
& A_{n}(z)=\prod_{b<-t_{n}} \tau\left(z ; b, \lambda_{b}\right)^{-1}=\prod \frac{a_{b}^{z}+a_{b}^{b}}{a_{b}^{z}-a_{b}^{b}}, \\
& B_{n}(z)=\prod_{-t_{n}<b<t_{n}} \tau\left(z ; b, n \lambda_{0}\right)=\prod \frac{4^{z / n}-4^{b / n}}{4^{z / n}+4^{b / n}}, \\
& C_{n}(z)=\prod_{b>t_{n}}\left(-\tau\left(z ; b, \lambda_{0}\right)^{-1}\right)=\prod \frac{4^{b}+4^{z}}{4^{b}-4^{z}}, \\
& D_{n}(z)=A_{n}(z) B_{n}(z) C_{n}(z) .
\end{aligned}
$$

LEMMA 6. (a) The product for $D_{n}$ converges normally on the plane to a meromorphic function all of whose zeros and poles are simple.

(b) $\left\{z: D_{n}(z)=0\right.$ or $\infty$ and $\left.|\operatorname{Im} z|<4 \lambda_{0} / 3\right\}=\mathscr{D}$. 
(c) If $D_{n}(z)=\infty$ and $|\operatorname{Re} z|<t_{n}$, then either $z \in \mathscr{D}$ or else $|\operatorname{Im} z|>2 n \lambda_{0}$. For every $\delta>0$ and $t>0$ the following hold for all large enough $n$.

(d) $\left|D_{n}(z)-1\right|<\delta$ whenever $t_{n}-t \leqslant|\operatorname{Re} z| \leqslant t_{n}+t$.

(e) $\left|D_{n}(z)\right|<1+\delta$ on $S_{n \lambda_{0}} \cap\left\{z:|\operatorname{Re} z| \leqslant t_{n}+t\right\}$.

(f) $\left|D_{n}(z)\right|>1-\delta$ on $\left(S_{\lambda_{0}} \cap\left\{z \mid \operatorname{Re} z \geqslant t_{n}-t\right\}\right) \cup\left(S_{\lambda_{0}-\delta} \cap\left\{z: \operatorname{Re} z \leqslant-t_{n}+\right.\right.$ $t\})$.

Proof. Let $\varepsilon$ be very small, let $x=\operatorname{Re} z$, and let $-s_{k}-1<b<-s_{k}<x-1$. By Lemma 2

$$
\left|1-\tau\left(z ; b, \lambda_{b}\right)^{-1}\right| \leqslant 4 \cdot 4^{(k+1) x / k} \cdot 4^{-(k+1) s_{k} / k} \leqslant 4 \cdot 4^{2 x} \cdot 4^{-s_{k}} \cdot 4^{-s_{k} / k} .
$$

From Lemma 1 and part (3) of Lemma 4 it follows that $A_{n}$ is normally convergent on $\mathrm{C}$ and that $\left|A_{n}(z)-1\right|<\varepsilon$ for $-t_{n}-t \leqslant \operatorname{Re} z$, for all large $n$. In a similar manner we find that $C_{n}$ is normally convergent and $\left|C_{n}(z)-1\right|<\varepsilon$ for $\operatorname{Re} z<t_{n}$ $+t$, for large $n . B_{n}$ is convergent, being a finite product, and Lemma 1, Lemma 2 , and part (2) of Lemma 4 give $\left|B_{n}(z)-1\right|<\varepsilon$ for $\operatorname{Re} z>t_{n}-t$, for large $n$. Because there are an even number of $b$ 's in $\left(-t_{n}, t_{n}\right), B_{n}(z)=\Pi \tau=\Pi(-\tau)$, and the same argument as above shows that $\left|B_{n}(z)-1\right|<\varepsilon$ for $|\operatorname{Re} z|>t_{n}-t$, for large $n$. Each $\tau(z ; b, \lambda)$ is periodic with period $4 \lambda i$, and $z_{0}$ is a zero of $\tau$ if and only if $z_{0}+2 \lambda i$ is a pole. The smallest $\lambda$ involved in the product for $D_{n}$ is $\lambda=$ $k \lambda_{0}(1+k)^{-1}$ for $k=n+1$; so $\lambda \geqslant 2 \lambda_{0} / 3$ and $2 \lambda \geqslant 4 \lambda_{0} / 3$. (a), (b), and (c) are clear, and if $\varepsilon$ is small enough (d) follows from the estimates above. Because $\left|A_{n}\right|$ and $\left|C_{n}\right|$ are each bounded by $1+\varepsilon$ in $|\operatorname{Re} z| \leqslant t_{n}+t$ and $\left|B_{n}\right| \leqslant 1$ in $S_{n \lambda_{0}}$, we have (e) for small $\varepsilon$ and large $n$. Finally, because $\left|B_{n}(z)-1\right|<\varepsilon$ for $|\operatorname{Re} z|>t_{n}-$ $t,\left|C_{n}\right| \geqslant 1$ in $S_{\lambda_{0}}$, and $\left|A_{n}\right| \geqslant 1$ in $S_{(n+1) \lambda_{0} /(n+2)}$, we have (f) for small $\varepsilon$ and large $n$.

LEMMA 7. If $z_{0} \in \mathbf{C}, \delta>0$, and $V$ is an open neighborhood of an arc which connects $z_{0}$ to $\infty$, then there exists an entire function $h$ having a simple zero at $z_{0}$ with no other zeros such that $|h-1|_{\mathrm{C}-V} \leqslant \delta$.

Proof. $\left|e^{\alpha}-1\right| \leqslant 2|\alpha|$ for $|\alpha| \leqslant 1$, by Lemma $1(b)$; so $|\alpha-\beta|<1$ implies $\left|e^{\alpha}-e^{\beta}\right| \leqslant 2|\alpha-\beta|\left|e^{\beta}\right|$. Choose a branch of $\log \left(z-z_{0}\right)^{-1}=-\log \left(z-z_{0}\right)$ in the complement of the given arc $\gamma$ which joins $z_{0}$ to $\infty$. In $V$ choose a connected simply connected neighborhood $V_{1}$ of $\gamma$ which is the interior of a locally polygonal set. Then $V_{1} \cup\{\infty\}$ is connected and locally connected; so Arakelyan's Theorem [A1], [A2] can be applied to the function $\log \left(z-z_{0}\right)^{-1}$ on $\mathbf{C}-V_{1}$ with $\varepsilon=$ $\min (1, \delta / 2)$ to yield an entire function $g$ so that $\left|g(z)-\log \left(z-z_{0}\right)^{-1}\right|<\varepsilon$ for $z \in \mathbf{C}-V_{1} \supseteq \mathbf{C}-V$. Put $\alpha=g(z)$ and $\beta=\log \left(z-z_{0}\right)^{-1}$ in the opening sentence of this proof, and we obtain $\left|\exp (g(z))-\left(z-z_{0}\right)^{-1}\right|<2 \varepsilon\left|z-z_{0}\right|^{-1}<\delta /\left|z-z_{0}\right|$ for $z \in \mathbf{C}-V$. Thus, $\left|\left(z-z_{0}\right) \exp (g(z))-1\right|<\delta$ for $z \in \mathbf{C}-V$. The function $h(z)=\left(z-z_{0}\right) \exp (g(z))$ has the desired behavior.

COROllary 8. There is a sequence $H_{n}$ of meromorphic functions on $\mathrm{C}$ which have these properties. Let $t>0$ and $\delta>0$ be arbitrary.

(1) $H_{n}(\bar{z})=\overline{H_{n}(z)}$.

(2) The zeros of $H_{n}$ are simple and comprise the set $\left\{b:|b|<t_{n}\right\}$.

(3) The poles of $H_{n}$ are simple and comprise the set $\left\{b:|b|>t_{n}\right\}$. 
(4) $\sup \left\{\left|H_{n}(z)-1\right|: t_{n}-t \leqslant|\operatorname{Re} z| \leqslant t_{n}+t\right\} \rightarrow 0$ as $n \rightarrow \infty$.

(5) $\sup \left\{\left|H_{n}(z)\right|:|\operatorname{Re} z| \leqslant t_{n}+t\right.$ and $\left.|\operatorname{Im} z| \leqslant n \lambda_{0}\right\} \rightarrow 1$ as $n \rightarrow \infty$.

(6) $\inf \left\{\left|H_{n}(z)\right|: \operatorname{Re} z \geqslant t_{n}-t\right.$ and $\left.|\operatorname{Im} z| \leqslant \lambda_{0}\right\} \rightarrow 1$ as $n \rightarrow \infty$.

(7) $\inf \left\{\left|H_{n}(z)\right|: \operatorname{Re} z \leqslant-t_{n}+t\right.$ and $\left.|\operatorname{Im} z| \leqslant \lambda_{0}-\delta\right\} \rightarrow 1$ as $n \rightarrow \infty$.

Proof. Start with the meromorphic functions $D_{n}$ of Lemma 6; note that $D_{n}(\bar{z})=\overline{D_{n}(z)}$. Enumerate the zeros and poles of $D_{n}$ in $\{z: \operatorname{Im} z>0\}$ as $z_{1}$, $z_{2}, \ldots ;$ then $\bar{z}_{1}, \bar{z}_{2}, \ldots$ are the zeros and poles in the lower half-plane. For each $j$ let $V_{j}$ be a small neighborhood of the line segment $L_{j}=\left\{z=x+i y: x=\operatorname{Re} z_{j}\right.$ and $\mathrm{y} \geqslant \operatorname{Im} z_{j}$ \} which joins $z_{j}$ to $\infty$. Because $L_{j}$ is disjoint from the set $T_{n}=S_{\lambda_{0}} \cup$ $\left\{z:|\operatorname{Re} z| \leqslant t_{n}\right.$ and $\left.|\operatorname{Im} z| \leqslant n \lambda_{0}\right\} \cup\left\{z:|\operatorname{Re} z| \in \cup\left[s_{k}, s_{k}+1\right]\right\}$, we may assume that $V_{j} \cap T_{n}=\varnothing$, as well. By Lemma 7 there is an entire function $h_{j}$ which is zero only at $z_{j}$ and which satisfies $\left|h_{j}-1\right| \leqslant \delta_{j}=2^{-n-j}$ outside $V_{j}$. Put $k_{j}(z)=\overline{h_{j}(\bar{z})}$. Let $F_{n}=\Pi\left(h_{j} k_{j}\right)^{ \pm 1}$, where the exponent is chosen to be +1 in case $D_{n}\left(z_{j}\right)=\infty=$ $D_{n}\left(\bar{z}_{j}\right)$ and is chosen to be -1 in case $D_{n}\left(z_{j}\right)=D_{n}\left(\bar{z}_{j}\right)=0$. Because $\sum 2 \delta_{j}=2 \cdot 2^{-n}$ $<\infty$ and each compact set meets only finitely many of the $V_{j}$ or their conjugates, the product for $F_{n}$ converges normally on the plane to a meromorphic function which by Lemma 1 satisfies $\left|F_{n}-1\right| \leqslant 62^{-n}$ on $T_{n}$, for $n \geqslant 2$. From Lemma 6 it is clear that $H_{n}=F_{n} D_{n}$ has all the required properties.

Define $R\left(x_{0} ; t, \lambda\right)$ to be the rectangle $\left\{z=x+i y:\left|x-x_{0}\right| \leqslant t\right.$ and $\left.|y|<\lambda\right\}$. For $n>0$ define $t_{-n}=-t_{n}, G_{n}(z)=2^{z-t_{n}} H_{n}(z)$, and $G_{-n}(z)=2^{t_{n}-z} H_{n}(z)$, where $H_{n}$ is as in Lemma 8. Fix $\lambda_{+} \leqslant \lambda_{0}$ and $\lambda_{-}<\lambda_{0}$, define $\lambda_{n}=\lambda_{+}$for $n>0$ and $\lambda_{n}=\lambda_{-}$ for $n<0$, and put $S(n)=S_{\lambda_{n}}^{+}$for $n>0$ and $S(n)=S_{\lambda_{n}}^{-}$for $n<0$. Define these sets:

$$
\begin{gathered}
\sigma_{n}=\left\{z \in S(n): 16^{-1} \leqslant\left|G_{n}(z)\right|<16\right\}, \\
X_{n}=\left\{z:|\operatorname{Re} z| \leqslant s_{|n|}+1 \text { and }|\operatorname{Im} z| \leqslant|n| \lambda_{0}\right\} \\
\cup\left(S(n) \cap\left\{z: \operatorname{Re} z / t_{n} \leqslant 1\right\}\right)-\sigma_{n}, \\
Y_{n}=\left(S(n) \cap\left\{z: \operatorname{Re} z / t_{n}>1\right\}\right)-\sigma_{n} .
\end{gathered}
$$

LEMMA 9. The following statements hold for $|n|$ sufficiently large.

(1) $G_{n}$ is meromorphic on $\mathbf{C}$ with a simple zero or pole at each $b \in \mathscr{D}$ and no other zeros nor poles; $G_{n}(\bar{z})=\overline{G_{n}(z)}$.

(2) $G_{n}$ is an analytic homeomorphism on a neighborhood of $R\left(t_{n} ; 5, \lambda_{n}\right)$.

(3) $R\left(t_{n} ; 3, \lambda_{n}\right) \subseteq \sigma_{n} \subseteq R\left(t_{n} ; 5, \lambda_{n}\right)$.

(4) $\left|G_{n}\right|<16^{-1}$ on $X_{n},\left|G_{n}\right|>16$ on $Y_{n}, 16^{-1} \leqslant\left|G_{n}\right| \leqslant 16$ on $\sigma_{n}$, and $G_{n}$ is an analytic homeomorphism on $\sigma_{n}$.

(5) There is a smooth function $\Theta_{n}:\left[16^{-1}, 16\right] \rightarrow(0, \pi)$ such that $G_{n}\left(\sigma_{n}\right)=\{w$ : $16^{-1}<|w| \leqslant 16$ and $\left.|\arg w| \leqslant \Theta_{n}(|w|)\right\}$.

Proof. For definiteness let us consider the case $n<0$; the case $n>0$ is treated in a very similar manner. (1) is clear because the same thing is true for $H_{n}$. By Corollary $8 H_{-n}\left(z+t_{n}\right) \rightarrow 1$ uniformly on $R\left(0 ; 7,3 \lambda_{0}\right)$ as $n \rightarrow-\infty$. Because $2^{-z}$ is an analytic homeomorphism on $S_{3 \lambda_{0}}$ and $G_{n}\left(z+t_{n}\right)=2^{-z} H_{-n}\left(z+t_{n}\right) \rightarrow 2^{-z}$ uniformly on $R\left(0 ; 7,3 \lambda_{0}\right), G_{n}\left(z+t_{n}\right)$ is an analytic homeomorphism on $R\left(0 ; 6,2 \lambda_{0}\right)$ for large $n<0$, and (2) follows. 
From parts (7) and (5) of Corollary 8 we obtain $\frac{1}{2}<\left|H_{-n}(z)\right|$ for $\operatorname{Re} z<t_{n}+5$ and $\left|H_{-n}(z)\right|<2$ for $\operatorname{Re} z \geqslant t_{n}-5$ for large $n<0$. If $\operatorname{Re} z<t_{n}-5$, we have $\left|2^{t_{n}-z}\right|>32$; so $\left|G_{n}(z)\right|>16$. If $\operatorname{Re} z>t_{n}+5$, we have $\left|2^{t_{n}-z}\right|<32^{-1}$; so $\left|G_{n}(z)\right|<$ $16^{-1}$. Thus, $\sigma_{n} \subseteq R\left(t_{n} ; 5, \lambda_{n}\right)$. Because $\frac{1}{2}<\left|H_{-n}(z)\right|<2$ in $R\left(t_{n} ; 5, \lambda_{n}\right)$, which contains $R\left(t_{n} ; 3, \lambda_{n}\right)$, and $8^{-1} \leqslant\left|2^{t_{n}-z}\right| \leqslant 8$ on $R\left(t_{n} ; 3, \lambda_{n}\right)$, we see that $16^{-1}<\left|G_{n}(z)\right|$ $\leqslant 16$ on $R\left(t_{n} ; 3, \lambda_{n}\right)$. Thus, $R\left(t_{n} ; 3, \lambda_{n}\right) \subseteq \sigma_{n}$ and (3) is proved.

Because $t_{|n|}-s_{|n|} \gg 0$ for large $|n|$, we have from Corollary 8 that $\left|H_{-n}(z)\right|<2$ for $z \in X_{n}$ for large $n<0$. By (3) and the definition of $X_{n}, \operatorname{Re} z \geqslant t_{n}+1$ for $z \in X_{n}$; so $\left|2^{t_{n}-z}\right| \leqslant \frac{1}{2}$ and $\left|G_{n}(z)\right|<2 \cdot \frac{1}{2}=1$ for $z \in X_{n}$. Because of (3) and the definition of $\sigma_{n}$, this means that $\left|G_{n}\right|<16^{-1}$ on $X_{n}$. In a similar manner we obtain $\left|G_{n}\right|>16$ on $Y_{n}$. By (2) and (3), $G_{n}$ is an analytic homeomorphism on $\sigma_{n}$, and (4) is proved.

Because $G_{n}$ is a diffeomorphism and does not vanish on a neighborhood of $R\left(t_{n} ; 5, \lambda_{n}\right)$, the functions $r=\left|G_{n}(z)\right|$ and $\theta=\arg \left(G_{n}(z)\right)$, where $-\pi<\theta<\pi$, constitute a global differentiable coordinate pair on a neighborhood of $R\left(t_{n} ; 5, \lambda_{n}\right)$. We can therefore parametrize $G_{n}\left(x-i \lambda_{n}\right)$ as $G_{n}\left(x-i \lambda_{n}\right)=r \exp \left[i \Theta_{n}(r)\right]$ for a smooth function $\Theta_{n}$. Because arg $2^{t_{n}-z}>0$ for $\operatorname{Im} z=-\lambda_{n}$ and $H_{-n}$ is nearly $1, \Theta_{n}$ takes values in $(0, \pi)$ for large $n<0$. The set $\gamma=\left\{r=r_{0}\right\} \cap R\left(t_{n} ; 5, \lambda_{n}\right)$ consists of regular arcs which have no endpoints in $R\left(t_{n} ; 5, \lambda_{n}\right)^{\circ}$. If we knew that $\gamma$ consisted of just one arc which meets the boundary of $R\left(t_{n} ; 5, \lambda_{n}\right)$ in just two points, we could complete the argument as follows. For $r_{0} \in\left[16^{-1}, 16\right] \gamma$ does not meet $\left\{x-t_{n}= \pm 5\right\}$, for on the latter set $\left|G_{n}\right|$ is approximately 32 or $32^{-1}$, which is not close to 16 or $16^{-1}$. So $\gamma$ connects $\operatorname{Im} z=-\lambda_{n}$ to $\operatorname{Im} z=+\lambda_{n}$. Because $G_{n}(\bar{z})=\overline{G_{n}(z)}$ and $G_{n}>0$ on $\left[t_{n}-5, t_{n}+5\right], \gamma$ is parametrized by a single symmetric interval $-\theta_{0} \leqslant \theta \leqslant \theta_{0}$. Evidently $\left(r_{0}, \theta_{0}\right)$ corresponds to a point of $\{\operatorname{Im} z=$ $\left.\lambda_{n}\right\}$ or to a point of $\left\{\operatorname{Im} z=-\lambda_{n}\right\}$. As we have previously observed, $\left\{\operatorname{Im} z=-\lambda_{n}\right\}$ corresponds to positive $\theta$. Since $G\left(x-i \lambda_{n}\right)=r \exp \left(i \Theta_{n}(r)\right)$, this shows that (5) holds.

Finally, to see that $\gamma=\left\{r=r_{0}\right\} \cap R\left(t_{n} ; 5, \lambda_{n}\right)$ consists of a single arc, consider the following elementary calculation for an analytic nonvanishing $f$ :

$$
\begin{aligned}
2|f| \frac{\partial|f|}{\partial x} & =\frac{\partial|f|^{2}}{\partial x}=\frac{\partial\left(f f^{-}\right)}{\partial x}=\frac{\partial f}{\partial x} \bar{f}+f \frac{\partial \bar{f}}{\partial x} \\
& =\bar{f} \frac{\partial f}{\partial x}+f\left(\frac{\partial f}{\partial x}\right)^{-}=\bar{f} f^{\prime}+f\left(f^{\prime}\right)^{-}=2 \operatorname{Re} \bar{f} f^{\prime}
\end{aligned}
$$

so $\partial|f| / \partial x=|f|^{-1} \operatorname{Re}\left(\bar{f} f^{\prime}\right)$. Apply this formula to $G_{n}$, taking into account the fact that $H_{-n}$ is very close to 1 on a neighborhood of $R\left(t_{n} ; 5, \lambda_{n}\right)$ and hence $H_{-n}^{\prime}$ is very close to 0 . The result is

$$
\begin{aligned}
\frac{\partial\left|G_{n}\right|}{\partial x} & =\left|G_{n}\right|^{-1} \operatorname{Re}\left(\bar{G}_{n} G_{n}^{\prime}\right) \approx\left|2^{t_{n}-z}\right|^{-1} \operatorname{Re}\left(\left(2^{t_{n}-z}\right)^{-}\left(2^{t_{n}-z}\right)^{\prime}\right) \\
& =\frac{\partial}{\partial x}\left|2^{t_{n}-z}\right|=(\log 2)\left|2^{t_{n}-z}\right| \leqslant-(\log 2) 32^{-1}
\end{aligned}
$$

on $R\left(t_{n} ; 5, \lambda_{n}\right)$. So for large negative $n, \partial r / \partial x=\partial\left|G_{n}\right| / \partial x<0$ on $R\left(t_{n} ; 5, \lambda_{n}\right)$. This means that each horizontal line $\{\operatorname{Im} z=$ constant $\}$ meets $\gamma$ in at most one point; 
and $\gamma$ consists of at most one arc, since it has no endpoints in $R\left(t_{n} ; 5, \lambda_{n}\right)^{\circ}$.

Define for large $|n|$ the following subsets of $\mathbf{C}$. [See Figure 1.]

$$
\begin{aligned}
V_{n}^{+} & =\left\{z: 4^{-1}<|z|<4 \text { and } \frac{1}{2} \Theta_{n}(|z|)<\arg z<\pi-\frac{1}{2} \Theta_{n}(|z|)\right\}, \\
V_{n}^{-} & =\left\{z: \bar{z} \in V_{n}^{+}\right\}, \\
W & =\left\{z: 4^{-1} \leqslant|z| \leqslant 4 \text { and }|\arg z| \leqslant \frac{1}{2} \Theta_{n}(|z|)\right\}, \\
W^{\prime} & =\{z:-z \in W\} .
\end{aligned}
$$

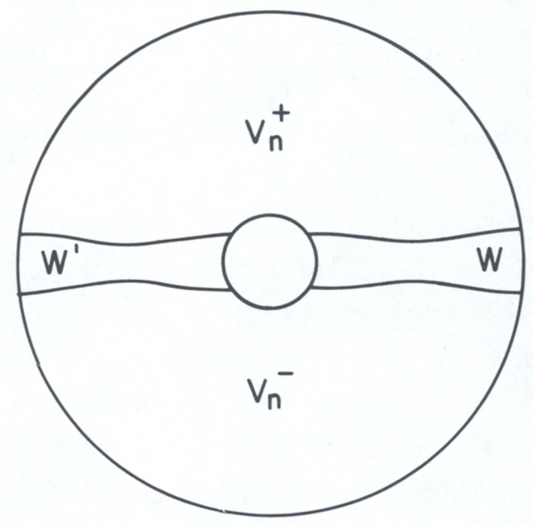

FIGURE 1
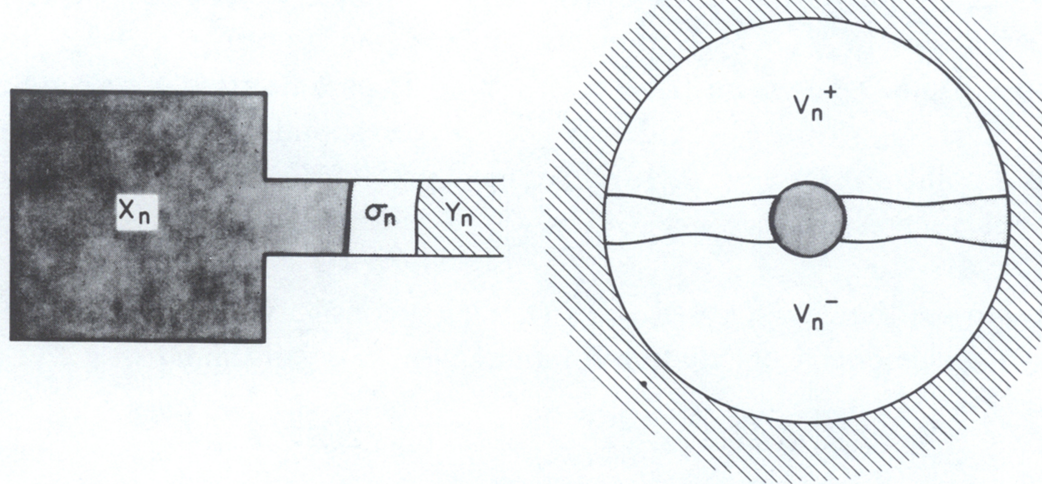

Figure 2

LEMma 10. For large $|n|$ there is an analytic function $\pi_{n}$ on $M$ which enjoys these properties [see Figure 2].

(1) $\pi_{n}^{2}=G_{n} \circ Z$;

(2) $\pi_{n}$ maps $Z^{-1}\left(X_{n} \cup \sigma_{n} \cup Y_{n}\right)$ into $\mathbf{C}-\left(V_{n}^{+} \cup V_{n}^{-}\right)$;

(3) $\pi_{n}\left(Z^{-1}\left(X_{n}\right)\right) \subseteq\left\{z:|z|<4^{-1}\right\}, \pi_{n}\left(Z^{-1}\left(Y_{n}\right)\right) \subseteq\{z:|z|>4\}$, and $\pi_{n}$ is an analytic homeomorphism of $Z^{-1}\left(\sigma_{n}\right)$ onto $W \cup W^{\prime}$.

Proof. Recall the surface $\bar{M}$ and its projection $\bar{\pi}$ onto $\mathrm{C}$, which has branch set $B \subseteq \bar{M} . G_{n}$ has a simple zero or pole at each point $b$ of $\mathscr{D}=\bar{\pi}(B)$, and no other zeros nor poles. From this fact and the definition of $\bar{M}$ and $\bar{\pi}$ it follows that the function $G_{n} \circ \bar{\pi}$ has a single-valued square root, call it $\bar{\pi}_{n}$, on $\bar{M}$. Indeed, $\bar{M}$ can be 
thought of as the classical Riemann surface constructed from the multiple-valued function $\sqrt{G_{n}}$ on the plane. Restricting $\bar{\pi}_{n}$ to $M$ we have $\sqrt{G_{n} \circ Z}$ as a single-valued analytic function, call it $\pi_{n}$, on $M$ having no zeros nor poles. Properties (2) and (3) are immediate from Lemma 9. Note that $\pi_{n}\left(p^{+}\right)=-\pi_{n}\left(p^{-}\right)$if $\left\{p^{+}, p^{-}\right\}=Z^{-1}(z)$ for $z \in \sigma_{n}$.

LeMma 11. Let $|n|$ be large. For every $\delta>0$ and every $C^{1}$ function $g$ on $Z^{-1}\left(X_{n} \cup \sigma_{n} \cup Y_{n}\right)$ such that $g=0$ on the closure of $Z^{-1}\left(X_{n} \cup Y_{n}\right)$ and $|\partial g / \partial \bar{Z}|<$ $\delta$ on $Z^{-1}\left(\sigma_{n}\right)$ there exists a meromorphic function $\psi$ on $M$ such that $|\psi-g|<17 \delta$ on $Z^{-1}\left(X_{n} \cup \sigma_{n} \cup Y_{n}\right)$. The poles of $\psi$ lie outside $Z^{-1}\left(X_{n} \cup \sigma_{n} \cup Y_{n}\right)$.

Proof. Let $|n|$ be so large that Lemma 10 holds. By Lemma 10 and the hypothesis on $g$ we can find a $C^{1}$ function $\tilde{g}$ on $C$ such that $\tilde{g}=0$ on $\{z$ : $|z|<4^{-1}+\varepsilon$ or $\left.|z|>4-\varepsilon\right\}$ for some $\varepsilon>0$ and $\tilde{g} \circ \pi_{n}=g$ on $Z^{-1}\left(X_{n} \cup \sigma_{n} \cup\right.$ $\left.Y_{n}\right)$. Calculate

$$
\frac{\partial g}{\partial \bar{Z}}=\frac{\partial\left(\tilde{g} \circ \pi_{n}\right)}{\partial \bar{Z}}=\frac{\partial\left(\tilde{g} \circ \pi_{n} \circ Z^{-1}\right)}{\partial \bar{z}} \circ Z=\frac{\partial \tilde{g}}{\partial \bar{z}} \circ \pi_{n} \circ Z^{-1} \circ Z=\frac{\partial \tilde{g}}{\partial \bar{z}} \circ \pi_{n} .
$$

So $|\partial \tilde{g} / \partial \bar{z}|<\delta$ on $W \cup W^{\prime}$. Now we apply a method of Mergelyan [M, \$3, Chapter I] to approximate $\tilde{g}$ by a rational function $k$. Then $k \circ \pi_{n}$ will approximate $\tilde{g} \circ \pi_{n}=g$. Let $\Gamma^{ \pm}$be curves oriented positively (counterclockwise) inside $V_{n}^{ \pm}$ which are within $\varepsilon$ of the boundary of $V_{n}^{ \pm}$. See Figure 3 .

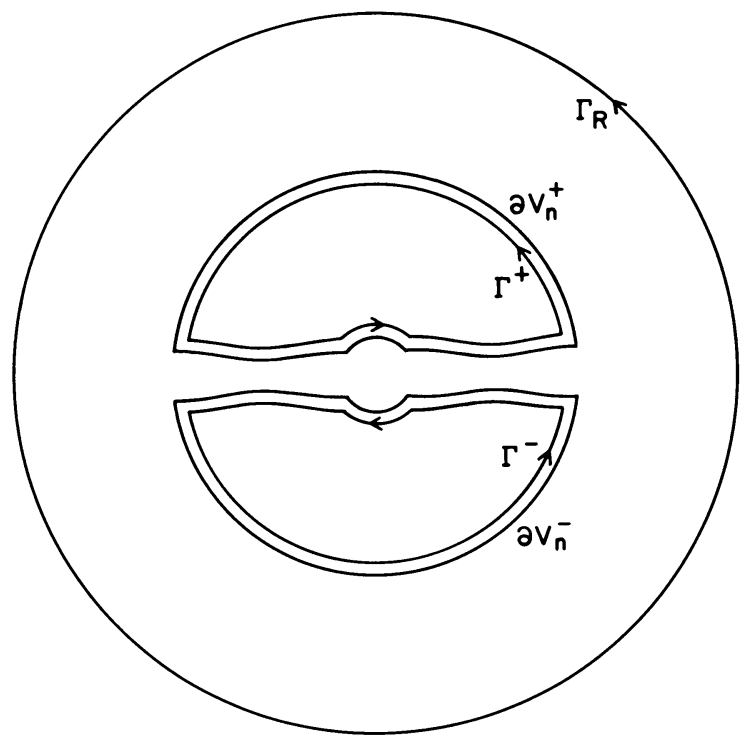

FIGURE 3

Let $\Gamma_{R}$ be the circle of radius $R$, centered at 0 . For $z_{0} \in \mathrm{C}-\left(V_{n}^{+} \cup V_{n}^{-}\right)$we can write the generalized Cauchy formula as follows (see [M, pp. 304-305], [G, p. 26], [S, 87]), for $R>\left|z_{0}\right|$.

$$
\tilde{g}\left(z_{0}\right)=\frac{1}{2 \pi i}\left(\int_{\Gamma_{R}}-\int_{\Gamma^{+} \cup \Gamma^{-}}\right) \tilde{g}(z)\left(z-z_{0}\right)^{-1} d z-\frac{1}{\pi} \iint_{\Sigma_{R}} \frac{\partial \tilde{g}}{\partial \bar{z}}\left(z-z_{0}\right)^{-1} d x d y,
$$


where $\Sigma_{R}$ is the set of points inside $\Gamma_{R}$ and outside both $\Gamma^{+}$and $\Gamma^{-}$. Because $\tilde{g}$ vanishes on $\Gamma_{R}$, the line integral reduces to

$$
I\left(z_{0}\right)=-(2 \pi i)^{-1} \int_{\Gamma^{+}} \cup \Gamma^{-} \tilde{g}(z)\left(z-z_{0}\right)^{-1} d z .
$$

Because $\tilde{g}$ vanishes on most of $\Sigma_{R}$, the integral over $\Sigma_{R}$ reduces to an integral over $T_{n}=$ the two components of $\left[\left\{4^{-1}+\varepsilon \leqslant|z|<4-\varepsilon\right\}-\left(\Gamma^{+} \cup \Gamma^{-}\right)\right]$which contain \pm 1 . See Figure 4 , in which $T_{n}$ is shaded.

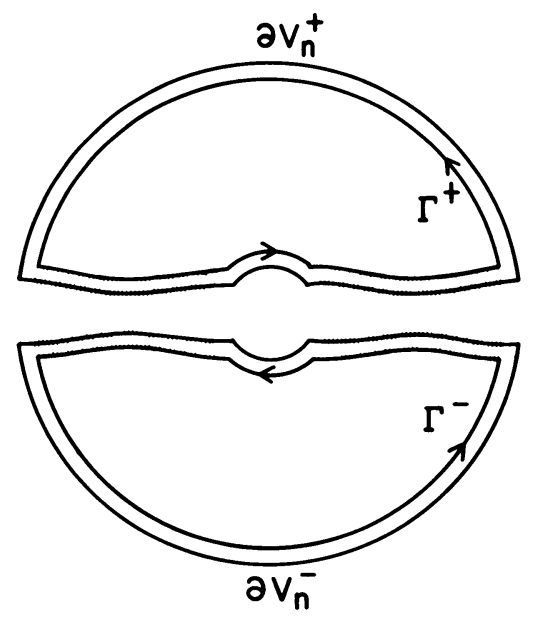

FiguRE 4

Because $\tilde{g}$ is $C^{1}$ and $|\partial \tilde{g} / \partial \bar{z}| \leqslant \delta$ on $C-\left(V_{n}^{+} \cup V_{n}^{-}\right)$, we can take $\varepsilon$ so small that $|\partial \tilde{g} / \partial \bar{z}|<2 \delta$ on $T_{n}$. Then

$$
\begin{aligned}
\left|\pi^{-1} \iint_{\Sigma_{R}}\right| & =\pi^{-1}\left|\iint_{T_{n}}\right| \leqslant 2 \delta \pi^{-1} \iint_{T_{n}}\left|z-z_{0}\right|^{-1} d x d y \\
& \leqslant 2 \delta \pi^{-1} \iint_{|z|<4}\left|z-z_{0}\right|^{-1} d x d y<2 \delta \pi^{-1} \sqrt{4 \pi \cdot \pi \cdot 4^{2}}=16 \delta
\end{aligned}
$$

The last inequality is Lemma 3.1.1 of [B]. Now for $z_{0} \in \mathbf{C}-\left(V_{n}^{+} \cup V_{n}^{-}\right)$and $z \in \Gamma^{+} \cup \Gamma^{-}$, the distance $\left|z-z_{0}\right|$ is bounded away from 0 ; thus, $I\left(z_{0}\right)$ can be uniformly approximated for $z_{0} \in \mathbf{C}-\left(V_{n}^{+} \cup V_{n}^{-}\right)$by a finite Riemann sum for this integral, which is manifestly a rational function $k\left(z_{0}\right)$ having its poles in $V_{n}^{+} \cup V_{n}^{-}$. Choosing such a $k$ for which $\left|I\left(z_{0}\right)-k\left(z_{0}\right)\right|<\delta$ for all $z_{0} \in \mathbf{C}-\left(V_{n}^{+}\right.$ $\left.\cup V_{n}^{-}\right)$, we have $|\tilde{g}-k| \leqslant 17 \delta$ on $\mathrm{C}-\left(V_{n}^{+} \cup V_{n}^{-}\right)$and so $\left|k \circ \pi_{n}-\tilde{g} \circ \pi_{n}\right|<$ $17 \delta$ on $\pi_{n}^{-1}\left(\mathrm{C}-\left(V_{n}^{+} \cup V_{n}^{-}\right)\right) \supseteq Z^{-1}\left(X_{n} \cup \sigma_{n} \cup Y_{n}\right)$. Thus, $\psi=k \circ \pi_{n}$ does what is required. The poles of $\psi$ are not in $Z^{-1}\left(X_{n} \cup \sigma_{n} \cup Y_{n}\right)$, because $\psi$ is bounded there.

LEMMA 12. Let $S$ be a closed subset of $\mathbf{C}$ which is star-shaped with respect to 0 , let $z_{n}$ be a sequence of points of $\mathrm{C}-S$ tending to $\infty$, and let $k_{n}$ be a sequence of nonnegative integers.f For each $\varepsilon>0$ there is an entire function $\varphi$ such that $|1-\varphi|<\varepsilon$ on $S$ and for each $n, \varphi$ has a zero of order at least $k_{n}$ at $z_{n}$.

Proof. Given $\varepsilon>0$, let $\varepsilon_{n}>0$ be chosen so that $\Sigma k_{n} \varepsilon_{n}<\min \left(\frac{1}{2}, \varepsilon / 3\right)$. Put $\gamma_{n}=\left\{r z_{n}: r \geqslant 1\right\} ; \gamma_{n}$ is an arc joining $z_{n}$ to $\infty$ in $\mathbf{C}-S$, because $S$ is star-shaped. 
Each compact set meets at most finitely many $\gamma_{n}$. Choose a neighborhood $V_{n}$ of each $\gamma_{n}$ so that $V_{n} \subseteq \mathrm{C}-S$ and so that each compact meets only finitely many $V_{n}$. Using Lemma 7 choose an entire function $h_{n}$ so that $h_{n}\left(z_{n}\right)=0$ and $\left|1-h_{n}\right|<\varepsilon_{n}$ on $\mathrm{C}-V_{n}$. By Lemma 1 the product $\Pi h_{n}^{k_{n}}$ converges normally on the plane to a function $\varphi$ having the desired properties.

COROLlary 13. In Lemma 11 we can require $\psi$ to be analytic on $M$ if we relax the approximation to $|\psi-g| \leqslant 18 \delta$.

Proof. Write $Z_{n}=Z^{-1}\left(X_{n} \cup \sigma_{n} \cup Y_{n}\right)$. Given $g$ and $\delta$ satisfying the hypothesis of Lemma 11, let $\psi_{1}$ be meromorphic on $M$ and satisfy $\left|\psi_{1}-g\right|_{Z_{n}}<17 \delta$. Let $P$ be the pole set of $\psi_{1}$ and enumerate $Z(P)=\left\{z_{1}, z_{2}, \ldots\right\}$. Note that $P \cap Z_{n}=\varnothing$; so $Z(P) \cap\left(X_{n} \cup \sigma_{n} \cup Y_{n}\right)=\varnothing$. For each $z_{j}$ let $k_{j}$ be the larger of the orders of the poles of $\psi_{1}$ at the two points of $Z^{-1}\left(z_{j}\right)$. In $Z_{n} g$ vanishes off a compact set; so $|g|_{Z_{n}}<\infty$. Thus $\left|\psi_{1}\right|_{Z_{n}}=K<\infty$. Apply Lemma 12 to the star-shaped $S=X_{n} \cup$ $\sigma_{n} \cup Y_{n}$, the sequence $\left\{z_{j}\right\}$, the integers $\left\{k_{j}\right\}$, and $\varepsilon=\delta / K$ to find an entire function $\varphi$ so that $|\varphi-1|_{s} \leqslant \delta / K$ and $\varphi$ has a zero of order at least $k_{j}$ at each $z_{j}$. Put $\psi=(\varphi \circ Z) \psi_{1}$, which has no poles on $M$ by construction.

$$
\left|\psi-\psi_{1}\right|_{Z_{n}}=\left|(\varphi \circ Z) \psi_{1}-\psi_{1}\right|_{Z_{n}} \leqslant\left|\psi_{1}\right|_{Z_{n}}|\varphi \circ Z-1|_{Z_{n}}<K \cdot \delta / K=\delta .
$$

Therefore, $|\psi-g|_{z_{n}} \leqslant\left|\psi-\psi \psi_{1}\right|_{z_{n}}+\left|\psi_{1}-g\right|_{z_{n}} \leqslant \delta+17 \delta=18 \delta$.

Equipped with the foregoing technical tools we can now detail the proof that $E_{\lambda}^{+} \in \mathbb{Q}$ for $\lambda \leqslant \lambda_{0}$ and $E_{\lambda}^{-} \in \mathbb{Q}$ for $\lambda<\lambda_{0}$. Fix one such $E_{\lambda}^{+}$or $E_{\lambda}^{-}$and call it $E$. Let $f \in A(E)$ and $\varepsilon>0$. By Corollary 13 there is a set of integers $\Re=\mathbf{Z} \cap$ $[N, \infty)$, in case $E=E_{\lambda}^{+}$, or $\Re=\mathbf{Z} \cap(-\infty,-N]$, in case $E=E_{\lambda}^{-}$, such that for every $n \in \mathcal{N}$ and every $C^{1}$ function $g$ on $Z_{n}=Z^{-1}\left(X_{n} \cup \sigma_{n} \cup Y_{n}\right)$ which is supported in the relative (to $Z_{n}$ ) interior of $Z^{-1}\left(\sigma_{n}\right)$ there is an analytic function $\psi$ on $M$ such that $|g-\psi|_{Z_{n}} \leqslant 18|\partial g / \partial \bar{Z}|_{Z_{n}}$. (If $\delta=|\partial g / \partial \bar{Z}|_{Z_{n}}>0$, this is Corollary 13; if $\delta=0$, then $g$ is analytic on the connected set $Z_{n}^{\circ}$, and since it vanishes on $Z^{-1}\left(X_{n}\right)$, it vanishes identically and we can take $\psi=0$.) For every $n \in \Re$ put $W_{n}=E \cap Z^{-1}\left\{z:\left|\operatorname{Re} z-t_{n}\right|<1\right\} . E-\cup_{n \in \Re} W_{n}$ consists of a sequence of separated closed connected subsets, which we may number $E_{n}, n \in \mathcal{R}$. Do this numbering so that $W_{n}$ sits between $E_{n}$ and $E_{n^{\prime}}$ where $\left|n^{\prime}\right|=|n|+1$. Each $E_{n}$ has a neighborhood of finite genus; indeed, the closure of $E_{n}$ in $\bar{M}$ is compact. We may select these neighborhoods to be disjoint from each other. By Theorem 1.5 of [S] there is an analytic function $\Phi$ on $M$ such that $|f-\Phi|_{E_{n}}<2^{-|n|} \theta$, where $\theta=\varepsilon / 40$. Put $g=f-\Phi$. Note that $|g|_{\cup E_{n}} \leqslant \theta / 2$.

Select a $C^{1}$ function $\chi: C \rightarrow[0,1]$ which depends only on $x=\operatorname{Re} z$ and which has these properties: $\chi \equiv 1$ on $[-1,1], \chi \equiv 0$ outside $(-2,2)$, and $|\partial \chi / \partial x|<2$. Then $|\partial \chi / \partial \bar{z}| \leqslant 1$. Put $\chi_{n}=\chi \circ\left(Z-t_{n}\right)$; then $\left|\partial \chi_{n} / \partial \bar{Z}\right|<1$ on $M$, and we may assume that $\chi_{m} \chi_{n} \equiv 0$ for $m$ and $n$ in $\Re, m \neq n$, because of the large gaps between $t_{m}$ and $t_{n}$ for large $|n|$. Define $g_{n}=\chi_{n} g$ for $n \in \mathcal{X} .\left|\Sigma g_{n}-g\right|_{E}<\theta / 2$ because of the following. For each $p$ there is an integer $m$ such that $\left(\Sigma g_{n}\right)(p)=g_{m}(p)$, because at most one term in $\Sigma g_{n}(p)$ is nonzero. Thus, $\left|\Sigma g_{n}(p)-g(p)\right|=\mid g_{m}(p)$ $-g(p)|=| \chi_{m}(p)-1|| g(p) \mid$. If $\chi_{m}(p)-1 \neq 0$, then $p \in \cup E_{n}$ and $|g(p)| \leqslant \theta / 2$, while $\left|\chi_{m}(p)-1\right| \leqslant 1$. 
In $E^{\circ}$ we calculate

$$
\frac{\partial g_{n}}{\partial \bar{Z}}=\frac{\partial\left(\chi_{n} g\right)}{\partial \bar{Z}}=\frac{\partial \chi_{n}}{\partial \bar{Z}} g+\chi_{n} \frac{\partial g}{\partial \bar{Z}}=\frac{\partial \chi_{n}}{\partial \bar{Z}} g,
$$

because $g \in A(E)$. Now $\left(\left(\partial \chi_{n} / \partial \bar{Z}\right) g\right)(p)=0$ unless $1<\left|\operatorname{Re} Z(p)-t_{n}\right|<2$, in which case $\left|\left(\partial x_{n} / \partial \bar{Z}\right)(p)\right| \leqslant 1$ and $|g(p)|<\max \left\{2^{-|m|} \theta: m \in \Re, m=n-1, n\right.$, or $n+1\}<2 \cdot 2^{-|n|} \theta$. Thus, the support of $g_{n}$ in $Z_{n}$ is contained in $R\left(t_{n} ; 2, \lambda\right)$, which belongs to the relative (to $Z_{n}$ ) interior of $\sigma_{n}$, by Lemma 9 , and $\left|\partial g_{n} / \partial \bar{Z}\right|_{z_{n}}<2$. $2^{-|n|} \boldsymbol{\theta}$.

Next we approximate $g_{n}$ on $Z_{n}$ by a $C^{1}$ function $h_{n}$ on $Z_{n}$. Specifically, let $s_{n}$ be the map of $\sigma_{n}$ into $C$ given by $s_{n}(z)=t_{n}+r_{n}\left(z-t_{n}\right)$, where $r_{n}<1$ and $r_{n}$ is close to 1 , and define $h_{n}$ by $h_{n}=g_{n}$ on $Z^{-1}\left(X_{n} \cup Y_{n}\right)$ and $h_{n}=\left(\left.Z\right|_{\Sigma}\right)^{-1} \circ s_{n} \circ\left(\left.Z\right|_{\Sigma}\right)$ for each component $\Sigma$ of $Z^{-1}\left(\sigma_{n}\right)$. Because $g_{n}$ vanishes on a neighborhood of $Z^{-1}\left(X_{n} \cup Y_{n}\right)$, the same will be true for $h_{n}$ if $r_{n}$ is close enough to 1 . In this case $h_{n}$ will be $C^{1}$ on $Z_{n}$ and $|\partial h / \partial \bar{Z}|_{Z_{n}}=r_{n}|\partial g / \partial \bar{Z}|_{Z_{n}}<|\partial g / \partial \bar{Z}|_{Z_{n}}<2 \cdot 2^{-|n|} \theta$. Because $Z^{-1}\left(\sigma_{n}\right)$ is compact we may take $r_{n}$ so close to 1 that $\left|g_{n}-h_{n}\right|_{Z^{-1}\left(\sigma_{n}\right)}<2^{-|n|} \theta$.

By Corollary 13 there is an analytic function $\psi_{n}$ on $M$ such that $\left|\psi_{n}-h_{n}\right|_{z_{n}}<18$ $\cdot 2 \cdot 2^{-|n|} \theta=36 \cdot 2^{-|n|} \theta$. Because $\Sigma_{n \in \Re} 2^{-|n|}<1$ and every compact set in $M$ is contained in all but perhaps finitely many of the $Z_{n}$, the sum $\Sigma \psi_{n}$ converges normally on $M$ to an analytic function $\Psi$. Let $F$ be $\Phi+\Psi$, which is analytic on $M$, and estimate

$$
\begin{aligned}
|F-f| & =\left|\sum \psi_{n}+\Phi-f\right| \\
& =\left|\sum\left(\psi_{n}-h_{n}\right)+\sum\left(h_{n}-g_{n}\right)+\left(\sum g_{n}-g\right)+(g+\Phi-f)\right| \\
& \leqslant \sum\left|\psi_{n}-h_{n}\right|+\sum\left|h_{n}-g_{n}\right|+\left|\sum g_{n}-g\right|+|g+\Phi-f| .
\end{aligned}
$$

On $E$ the first sum is at most $\Sigma_{n \in \Re} 36 \cdot 2^{-|n|} \theta<36 \theta$. The second sum has at most one nonzero term at any point of $E$; so it is dominated by $\max \left\{2^{-|n|} \theta: n \in \Re\right\}<$ $\theta / 2$. The third term $\left|\sum g_{n}-g\right|$ is at most $\theta / 2$, as estimated earlier, and the last expression $|g+\Phi-f|$ is identically zero by definition of $g$. Therefore, we have $|F-f| \leqslant 37 \theta=37 \varepsilon / 40<\varepsilon$ on $E$, and the proof is complete.

\section{REFERENCES}

[A1] N. U. Arakelyan, Uniform approximation on closed sets by entire functions, Izv. Akad. Nauk SSSR Ser. Mat. 28 (1964), 1187-1206. (Russian)

[A2] _ Approximation complexe et propriétés des fonctions analytiques, Internat. Congr. Math., vol. 2, Gauthier-Villars, Paris, 1971, pp. 595-600.

[B] A. Browder, Introduction to function algebras, Benjamin, New York, 1969.

[BS] H. Behnke and F. Sommer, Theorie der analytischen Funktionen eines komplexen veranderlichen, 2nd ed., Springer-Verlag, Berlin and New York, 1962, pp. 581-592.

[G] T. W. Gamelin, Uniform algebras, Prentice-Hall, Englewood Cliffs, N. J., 1969.

[M] S. N. Mergelyan, Uniform approximations to functions of a complex variable, Amer. Math. Soc. Transl. (1) 3 (1964), 294-391.

[RS] B. Rodin and L. Sario, Principal functions, Van Nostrand, Princeton, N. J., 1968, p. 205.

[S] S. Scheinberg, Uniform approximation by functions analytic on a Riemann surface, Ann. of Math. 108 (1978), 257-298.

Department of Mathematics, University of California, Irvine, California 92717 